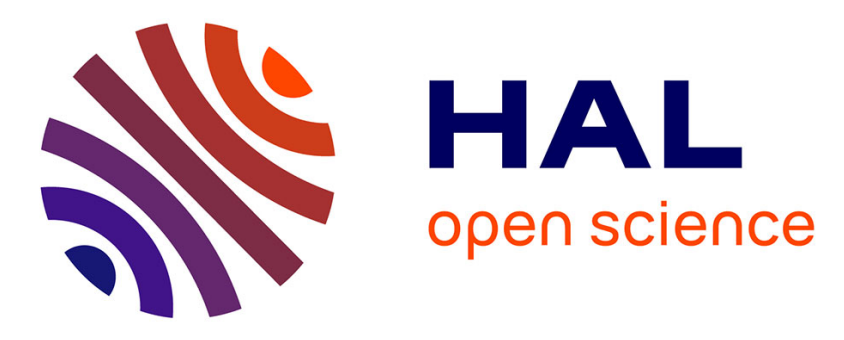

\title{
Ascending thoracic aorta aneurysm repair induces positive hemodynamic outcomes in a patient with unchanged bicuspid aortic valve
}

Francesca Condemi, Salvatore Campisi, Magalie Viallon, Pierre Croisille, Jean-François Fuzelier, Stéphane Avril

\section{To cite this version:}

Francesca Condemi, Salvatore Campisi, Magalie Viallon, Pierre Croisille, Jean-François Fuzelier, et al.. Ascending thoracic aorta aneurysm repair induces positive hemodynamic outcomes in a patient with unchanged bicuspid aortic valve. Journal of Biomechanics, 2018, 81, pp.145-148. hal-02004894

\section{HAL Id: hal-02004894 \\ https://hal.science/hal-02004894}

Submitted on 2 Feb 2019

HAL is a multi-disciplinary open access archive for the deposit and dissemination of scientific research documents, whether they are published or not. The documents may come from teaching and research institutions in France or abroad, or from public or private research centers.
L'archive ouverte pluridisciplinaire HAL, est destinée au dépôt et à la diffusion de documents scientifiques de niveau recherche, publiés ou non, émanant des établissements d'enseignement et de recherche français ou étrangers, des laboratoires publics ou privés. 


\title{
Ascending thoracic aorta aneurysm repair induces positive
}

\section{hemodynamic outcomes in a patient with unchanged}

\section{bicuspid aortic valve}

\author{
Francesca Condemia ${ }^{\text {, Salvatore Campisi }}{ }^{\mathrm{b}}$, Magalie Viallon ${ }^{\mathrm{b}, \mathrm{c}}$, Pierre Croisille $\mathrm{e}^{\mathrm{b}, \mathrm{c}}$, Jean-François \\ Fuzelier $^{\mathrm{b}}$, Stéphane Avrila \\ ${ }^{a}$ Mines Saint-Etienne, Univ Lyon, Univ Jean Monnet, INSERM, U 1059 Sainbiose, Centre CIS, F - 42023 Saint- \\ Etienne France; ${ }^{\mathrm{b}} \mathrm{CHU}$ Hôpital Nord, Saint-Etienne, France ; ${ }^{\mathrm{C}}$ Univ Lyon, UJM-Saint-Etienne, INSA, CNRS UMR \\ 5520, INSERM U1206, CREATIS, F-42023, Saint-Etienne, France
}

Short title: Positive hemodynamic outcome in a patient with BAV

\section{Short Communication}
Corresponding author: Stéphane Avril Univ Lyon, INSERM U1059,
Mines Saint-Etienne, SAINBIOSE, F-42023, 158 cours Fauriel, 42023 SAINT-ETIENNE cedex 2, France
Phone: +33477420188
Fax : +33477420000
Email: avril@emse.fr

Key words: Ascending Thoracic Aortic Aneurysms, Bicuspid Aortic Valve, 4D MRI, Computational Fluid Dynamics

Word count: 1994 words 
Abstract: We report a patient-specific case of bicuspid aortic valve with fusion of right and left coronary leaflets (R-L type I BAV), moderate aortic valve deficiency and ascending thoracic aortic aneurysms (ATAA) who was treated by only ascending aorta replacement preserving the BAV. The flow eccentricity, the helicity intensity $\left(\mathrm{h}_{2}\right)$, the circumferential time averaged wall shear stress

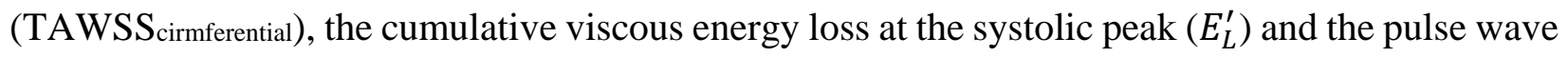
velocity (PWV) were calculated by combining 4D flow MRI and CFD analysis before (Stage I) and after (Stage II) the surgical procedure. CFD analyses assumed rigid walls, a non-Newtonian behavior for the blood and MRI measured patient-specific blood flow profiles as inlet boundary conditions. Stage II results showed suppression of recirculation in the ascending aorta, loss of jet

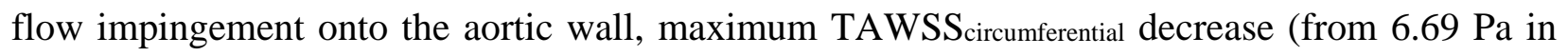
Stage I to $6 \mathrm{~Pa}$ in Stage II), reduction of flow helicity (from 10.97 in Stage I to $=8.47$ in Stage II) and $E_{L}^{\prime}$ (from $15.8 \mathrm{~mW}$ in Stage I to $11.2 \mathrm{~mW}$ in Stage II). However, Floweccentricity and PWV were found higher in Stage II due to the diameter reduction (Floweccentricity $=0.60$ in Stage I and Floweccentricity=0.91 in Stage II; PWV=3.80 m/s in Stage I m/s and PWV=9.37 m/s in Stage I).

Our work has permitted to compute for the first time the hemodynamic alterations obtained after restoration of normal ascending aorta and sinotubular junction geometry even preserving an R-L type I BAV with still acceptable function.

\section{Introduction}


Bicuspid aortic valve (BAV) is the most common congenital heart defect affecting $0.5-2 \%$ of general population and it is responsible for an increased risk of developing aortic valve and ascending thoracic aorta complications. Aortopathy with possible ascending thoracic aortic aneurysm (ATAA) formation and dissection accounts for the elevated cardiovascular mortality of patients with BAV (Hiratzka et al., 2010).

In case of normal or functionally acceptable BAV and supracoronary ATAA it remains under debate whether the aneurysm repair with restoration of sinotubular junction is itself the optimal surgical correction or the BAV prophylactic replacement is required (Hiratzka et al., 2010; Nishimura et al., 2014). 4D flow MRI studies have shown that BAV patients can have drastically altered hemodynamics with increased peak velocity, increased wall shear stress (WSS) and increased energetic load placed on the left ventricle. This altered hemodynamics has been related to aortopathy through mechanical weakening of the aortic wall. It is also known that patients with dilated ascending thoracic aorta, but with physiologically normal aortic valve, exhibit alterations in 3D aortic blood flow patterns such as enhanced helix or vortex flow, jet flow impingement and increased WSS (Barker et al., 2012; Bürk et al., 2012; Condemi et al., 2017).

BAV patients with dilated aorta may present a dilemma as their altered aortic hemodynamics may result from both the valve and the dilation, with no clue on how each lesion contributes to the effects.

We report a patient-specific case of R-L type I BAV with moderate aortic valve deficiency and ATAA who was treated by only ascending aorta replacement preserving the BAV. We used a combination of 4D MRI and computer fluid dynamics (CFD) models to show that a positive hemodynamic outcome can still be obtained in this patient only by reestablishing the normal ascending aorta geometry through aneurysm repair.

\section{Materials and methods}


The case study is a 59-year-old patient who presented R-L type I BAV, moderate aortic valve insufficiency and a $6.03 \mathrm{~cm} \times 5.95 \mathrm{~cm}$ ATAA (Figure 1). The patient underwent supracoronary ascending aorta replacement with a $32 \mathrm{~cm}$ Dacron polyester fabric graft and native aortic valve preservation (David et al., 2002).

We combined the use of 4D flow MRI and CFD analysis (with rigid walls) due to their unique complimentary advantages and limitations. Validated CFD models allow accurate analysis in terms of high temporal and spatial resolution which is particularly important to properly estimate the wall shear stress distribution. On the other hand, 4D flow MRI directly measures in vivo the 3D blood flow velocities independently of boundary conditions (Bürk et al., 2012; Condemi et al. 2017).

The study was approved by the Institutional Review Board of the University Hospital Center of Saint-Etienne. After providing informed consent, the patient was scanned before the surgery (Stage I) and 3 months after the surgery (Stage II) on a 3T MR scanner (Siemens Magnetom Prisma) without contrast agent (Strecker et al., 2012). The 4D flow MRI data analysis and visualization was performed using the cvi42@ prototype 4DFlow module (cmr ${ }^{42}$, Circle Cardiovascular Imaging Inc., Calgary, Canada) (Childs et al., 2011).

CFD analyses were performed using patient-specific blood flow profiles as inlet boundary condition (BC), velocity waveforms as outlet BCs at the apico-aortic vessels and a Windkessel model as BC at the descending aorta (Morbiducci et al., 2013; Youssefi et al., 2017). A tetrahedral mesh was built and mesh independency of the solution was reached for element size of $1 \mathrm{~mm}$ corresponding to a relative error in terms of velocity and pressure smaller than $2 \%$. The flow was assumed laminar, the aortic wall rigid, the blood incompressible and non-Newtonian according to the Carreau-Yasuda model (Shibeshi and Collins, 2005). The solution was obtained with Ansys Fluent v17.2 using the Semi-Implicit Method for Pressure-Linked Equations (SIMPLE), a secondorder interpolation scheme and a second-order upwind interpolation. A second order implicit time 
advanced scheme was used as transient-time solver and a time step of $1 \mathrm{~ms}$ was chosen. Three cardiac cycles were simulated. The convergence of the solution was assessed for residual errors below $10^{-3}$ (Condemi et al. 2017). After deriving blood flow pathlines at systole from CFD analysis, the following quantities were derived across the ascending thoracic aorta: the flow eccentricity (Floweccentricity, 0 indicates that the flow is centrally distributed with respect to the vessel centerline, and 1 indicates that the flow is eccentric and impinges against the vessel walls), the helicity

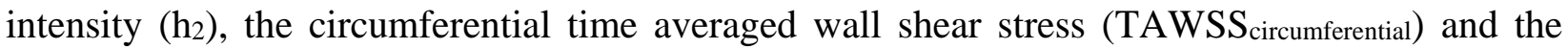
cumulative viscous energy loss at the systolic peak $\left(E_{L}^{\prime}\right)$. The pulse wave velocity (PWV) was calculated from the 4D MRI (Allen et al., 2013; Condemi et al., 2017; Gallo et al., 2012; Morbiducci et al., 2008; Sigovan et al., 2011).

\section{Results}

Stage I. 4D flow MRI and CFD analyses show Floweccentricity in the region of the bulge and downstream the region of the aneurysm. Floweccentricity in the mid ascending thoracic aorta was equal to 0.6 indicating the offset of the highest velocity flow away from the aortic centerline and blood flow impingement against the antero-lateral wall of the ascending thoracic aorta with a velocity peak of $1.65 \mathrm{~m} / \mathrm{s}$ (Figure 2A \& Figure 2B). TAWSS peak calculated from the CFD analysis was extended in the antero-lateral region of the aortic wall with a maximum value of 6.69 $\mathrm{Pa}$ (Figure 2C). Results also show h2 value equals to 10.97. Recirculation regions are visible in the most dilated part of the ascending thoracic aorta. The $E_{L}^{\prime}$ was of $15.8 \mathrm{~mW}$ and the PVW of $3.80 \mathrm{~m} / \mathrm{s}$.

Stage II. A significant decrease of $\mathrm{h}_{2}$ was found with the maximum value equals to 8.47. Recirculation regions were removed from the proximal part of the ascending thoracic aorta, but other regions of moderate recirculation appeared in the distal part just after the graft suture (Figure 3A \& Figure 3B). The major effect is a dramatic reorientation of velocity vectors in the aortic root. 
Whereas velocity vectors made an angle of about $45^{\circ}$ with the aortic centerline before the surgery, they recovered a direction nearly parallel to the centerline after the surgery. Consequently, $E_{L}^{\prime}$ was decreased to $11.2 \mathrm{~mW}$ after surgical repair.

The analysis also revealed unevenly distributed blood flow due to the presence of the BAV. The peak of velocity in the middle region of the graft $(1.83 \mathrm{~m} / \mathrm{s}$, Figure $3 \mathrm{~A} \&$ Figure $3 \mathrm{~B})$ was increased due to the reduction of diameter. Floweccentricity was of 0.91 . High TAWSS equals to $6 \mathrm{~Pa}$ was found in the region after the nozzle where helical flow was developed (Figure 3C). Moreover, the 4D MRI showed an increase of the PVW $(9.37 \mathrm{~m} / \mathrm{s})$.

\section{Patient follow-up information}

Six months after surgery, the patient was in class NYHA I (No limitation of physical activity). The follow-up echocardiography found an excellent left ventricular function, a trivial aortic insufficiency in the absence of aortic valve gradient and a good restoration of the synotubular junction diameter.

\section{Discussion}

BAV is a really complex spectrum of congenital heart defect and in a wider way it should be considered as a possible expression of the left ventricular outflow tract (LVOT) defects. Nearly $35 \%$ of BAV patients develop aortic valve and ascending aortic pathologies. The correlation between BAV phenotype and ATAA patterns is very complex and has led to formulate two main theories: the genetic and the hemodynamic one. The latter is recent and has benefited from 4D flow MRI studies which have shown how even normally functioning BAV have abnormal transvalvularflow patterns that cause regional increase of aortic WSS. Further, it has been possible to correlate BAV phenotype and different patterns of WSS distribution so that in the right-left cusp fusion BAV 
(R-L type I BAV), the flow is directed to the right anterior wall of the ascending aorta resulting in dilatation of this portion, while, in BAV with right-non coronary fusion (R-NC type I BAV), the flow is directed to the posterior wall resulting in proximal aortic arch dilatation. The change of TAWSS may have effects on cells which would deserve further dedicated study (Davies, 2009; Humphrey, 2002; Michel et al., 2018).

In this paper we showed a case where moderately deficient BAV were not replaced during the ATAA repair. Despite the persistence of aortic valve deficiency, the classical positive hemodynamic outcomes of supracoronary ascending aorta repair was still observed with a partial suppression of recirculation, loss of jet flow impingement onto the aortic wall, reduction of helicity intensity and $E_{L}^{\prime}$. Some of these positive hemodynamic outcomes related to geometric modifications of the ascending thoracic aorta were earlier reported by different authors but this unique case allow us to evaluate the real contribution on aortic hemodynamics of the restoration of normal ascending aorta geometry in presence of unchanged R-L type I BAV (Allen et al., 2013). Previous studies comparing bicuspid and tricuspid aortic valve patients after valve sparing aortic root replacement observed that abnormalities in aortic hemodynamics is not strictly related to the BAV phenotype but is more related to aortic blood flow and hemodynamics (Markl et al., 2005). According to these results, we submit that significant hemodynamic improvements could be expected also in other cases of ATAA. Most of the hemodynamic corrections can be explained by the reorientation of velocity vectors, as the inclination of velocity vectors is a major determinant of altered hemodynamics in dilated ascending thoracic aortas.

However, persistence of the BAV morphology did not permit to have significant changes of Floweccentricity at Stage II. The flow jet related to the BAV morphology persisted, but its impingement onto the wall was removed. We also found an increase in postoperative velocities due to the smaller diameter of the Dacron aortic graft. In addition, as previously discussed by 
others, 4D MRI showed an increase of PWV due to the decreased graft compliance compared with the aortic wall. This reduced elasticity may trigger end-organ damage due to peripheral hypertension. Reduction of the blood flow in the vascular bed during diastole due to the decreased Windkessel effect was also reported (Allen et al., 2013). These negative effects due to different mechanical properties of Dacron grafts used for ATAA correction still need to be investigated further and their clinical significance need to be evaluated versus the positive effects mentioned previously.

Limitations. In this study, the aortic walls were assumed rigid considering that the relatively stiff walls in the region of interest (ATAA) make their motion be rather small. We plan to achieve further analysis of these effects.

Direct numerical simulations (DNS) would be required to properly resolve turbulence. However, it was previously reported that the TAWSS distribution between high resolution (HR) and Normal Resolution (NR) simulations is comparable (Valen-Sendstad and Steinman, 2014).

\section{Conclusion}

It is still unclear how the thoracic aorta deranged hemodynamics may play a role in the pathogenesis of the aortic dilatation. Therefore, a better understanding of ascending aortic hemodynamics related to BAV could help clinicians for developing further personalized risk assessment for aortic complications in BAV patients. The unique combination of 4D flow MRI and CFD is a promising tool to contribute to this goal. It has permitted to compute for the first time the hemodynamic alterations obtained after restoration of normal ascending aorta and sinotubular junction geometry even preserving an R-L type I BAV with still acceptable function. As up to $2 \%$ of the population is affected by BAV, similar clinical cases should be analyzed in the future to complete and validate the very unique observations reported in the current case study. 


\section{Acknowledgments}

We thank Dr A.J. Barker and Dr M. Markl from Northwestern University of Chicago (IL, USA) who provided insight and expertise for the 4D MRI data analysis. We thank Dr Morbiducci and Dr Gallo from Polytechnic of Turin (Italy) who provided insight and expertise that greatly assisted the CFD modelling and data analysis. We are also grateful to Ansys, Inc. for providing AnsysFluent (ANSYS® Academic Research, Release 17.2).

This research was supported by the European Research Council (ERC grant biolochanics, grant number 647067).

\section{Conflict of interest statement}

The authors confirm there are not conflict of interest associated with this publication.

\section{References}

Allen, B.D., Barker, A.J., Kansal, P., Collins, J.D., Carr, J.C., Malaisrie, S.C., Markl, M., 2013. Impact of Aneurysm Repair on Thoracic Aorta Hemodynamics. Circulation 128, e341-e343.

Barker, A.J., Markl, M., Bürk, J., Lorenz, R., Bock, J., Bauer, S., Schulz-Menger, J., von KnobelsdorffBrenkenhoff, F., 2012. Bicuspid Aortic Valve Is Associated With Altered Wall Shear Stress in the Ascending Aorta. Circulation: Cardiovascular Imaging 5, 457-466.

Bürk, J., Blanke, P., Stankovic, Z., Barker, A., Russe, M., Geiger, J., Frydrychowicz, A., Langer, M., Markl, M., 2012. Evaluation of 3D blood flow patterns and wall shear stress in the normal and dilated thoracic aorta using flow-sensitive 4D CMR. Journal of Cardiovascular Magnetic Resonance 14, 84-84.

Childs, H., Ma, L., Ma, M., Clarke, J., Cocker, M., Green, J., Strohm, O., Friedrich, M.G., 2011. Comparison of long and short axis quantification of left ventricular volume parameters by cardiovascular magnetic resonance, with ex-vivo validation. Journal of Cardiovascular Magnetic Resonance 13, 40. 
Condemi, F., Campisi, S., Viallon, M., Troalen, T., Xuexin, G., Barker, A.J., Markl, M., Croisille, P., Trabelsi, O., Cavinato, C., Duprey, A., Avril, S., 2017. Fluid- and Biomechanical Analysis of Ascending Thoracic Aorta Aneurysm with Concomitant Aortic Insufficiency. Annals of Biomedical Engineering, 1-12.

David, T.E., Ivanov, J., Armstrong, S., Feindel, C.M., Webb, G.D., 2002. Aortic valve-sparing operations in patients with aneurysms of the aortic root or ascending aorta. The Annals of Thoracic Surgery 74, S1758S1761.

Davies, P.F., 2009. Hemodynamic shear stress and the endothelium in cardiovascular pathophysiology. Nature clinical practice. Cardiovascular medicine 6, 16-26.

Gallo, D., Steinman, D.A., Bijari, P.B., Morbiducci, U., 2012. Helical flow in carotid bifurcation as surrogate marker of exposure to disturbed shear. Journal of Biomechanics 45, 2398-2404.

Hiratzka, L.F., Bakris, G.L., Beckman, J.A., Bersin, R.M., Carr, V.F., Casey, D.E., Eagle, K.A., Hermann, L.K., Isselbacher, E.M., Kazerooni, E.A., Kouchoukos, N.T., Lytle, B.W., Milewicz, D.M., Reich, D.L., Sen, S., Shinn, J.A., Svensson, L.G., Williams, D.M., 2010. 2010 ACCF/AHA/AATS/ACR/ASA/SCA/SCAI/SIR/STS/SVM Guidelines for the Diagnosis and Management of Patients With Thoracic Aortic Disease. A Report of the American College of Cardiology Foundation/American Heart Association Task Force on Practice Guidelines, American Association for Thoracic Surgery, American College of Radiology, American Stroke Association, Society of Cardiovascular Anesthesiologists, Society for Cardiovascular Angiography and Interventions, Society of Interventional Radiology, Society of Thoracic Surgeons, and Society for Vascular Medicine 121, e266-e369.

Humphrey, J., 2002. Cardiovascular solid mechanics : cells, tissues, and organs. Springer, New York.

Michel, J.-B., Jondeau, G., Milewicz, D.M., 2018. From genetics to response to injury: vascular smooth muscle cells in aneurysms and dissections of the ascending aorta. Cardiovascular Research 114, 578-589. Morbiducci, U., Ponzini, R., Rizzo, G., Cadioli, M., Esposito, A., De Cobelli, F., Del Maschio, A., Montevecchi, F.M., Redaelli, A., 2008. In Vivo Quantification of Helical Blood Flow in Human Aorta by Time-Resolved Three-Dimensional Cine Phase Contrast Magnetic Resonance Imaging. Annals of Biomedical Engineering 37, 516.

Nishimura, R.A., Otto, C.M., Bonow, R.O., Carabello, B.A., Erwin, J.P., Guyton, R.A., O'Gara, P.T., Ruiz, C.E., Skubas, N.J., Sorajja, P., Sundt, T.M., Thomas, J.D., 2014. 2014 AHA/ACC Guideline for the Management of Patients With Valvular Heart Disease: Executive Summary. A Report of the American College of Cardiology/American Heart Association Task Force on Practice Guidelines 129, 2440-2492.

Shibeshi, S.S., Collins, W.E., 2005. The Rheology of Blood Flow in a Branched Arterial System. Applied rheology (Lappersdorf, Germany : Online) 15, 398-405.

Sigovan, M., Hope, M.D., Dyverfeldt, P., Saloner, D., 2011. Comparison of four-dimensional flow parameters for quantification of flow eccentricity in the ascending aorta. Journal of Magnetic Resonance Imaging 34, 1226-1230.

Strecker, C., Harloff, A., Wallis, W., Markl, M., 2012. Flow-sensitive 4D MRI of the thoracic aorta: Comparison of image quality, quantitative flow, and wall parameters at $1.5 \mathrm{~T}$ and $3 \mathrm{~T}$. Journal of Magnetic Resonance Imaging 36, 1097-1103.

Valen-Sendstad, K., Steinman, D.A., 2014. Mind the Gap: Impact of Computational Fluid Dynamics Solution Strategy on Prediction of Intracranial Aneurysm Hemodynamics and Rupture Status Indicators. American Journal of Neuroradiology 35, 536-543. 


\section{Figures Legends}

Figure 1. Lateral view of the ATAA 3D model (A); axial view of the ATAA (B).

Figure 2. Stage I: pathlines and velocity distribution in the region of the dilatation obtained, respectively, from the 4D MRI data (A) and the CFD study (B); TAWSS distribution obtained from the CFD study. The dashed line shows the highest TAWSS which was found in the antero-lateral region of the aortic wall with a maximum value of $6.69 \mathrm{~Pa}(\mathrm{C})$.

Figure 3. Stage II: pathlines and velocity distribution along and downstream the prosthesis obtained, respectively, from the 4D MRI data (A) and the CFD study (B); TAWSS distribution 
obtained from the CFD study. The dashed line shows the highest TAWSS which was found in the region after the nozzle with a maximum value of $6 \mathrm{~Pa}(\mathrm{C})$.

\section{Table}

Table 1. Pre-operative and post-operative results

\begin{tabular}{|l|c|c|c|c|c|c|}
\hline & $\begin{array}{c}\text { Flow } \\
\text { [4D MRI] }\end{array}$ & $\begin{array}{c}\text { Flow }_{\text {eccentricity }} \\
\text { [CFD] }\end{array}$ & $\mathbf{h}_{\mathbf{2}}$ & $\begin{array}{c}\text { TAWSS }_{\text {circumferential }} \\
{[\mathbf{P a}]}\end{array}$ & $\begin{array}{c}\boldsymbol{E}_{\boldsymbol{L}}^{\prime} \\
{[\mathbf{m W}]}\end{array}$ & $\begin{array}{c}\mathbf{P V W} \\
{[\mathbf{m} / \mathbf{s}]}\end{array}$ \\
\hline Stage I & 0.67 & 0.60 & 10.97 & 6.69 & 15.8 & 3.80 \\
\hline Stage II & 1.00 & 0.91 & 8.47 & 6.00 & 11.2 & 9.37 \\
\hline
\end{tabular}

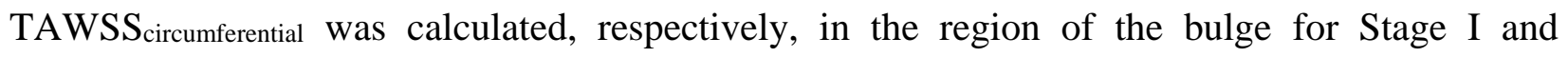
downstream the prosthesis for Stage II. 
Figure 1

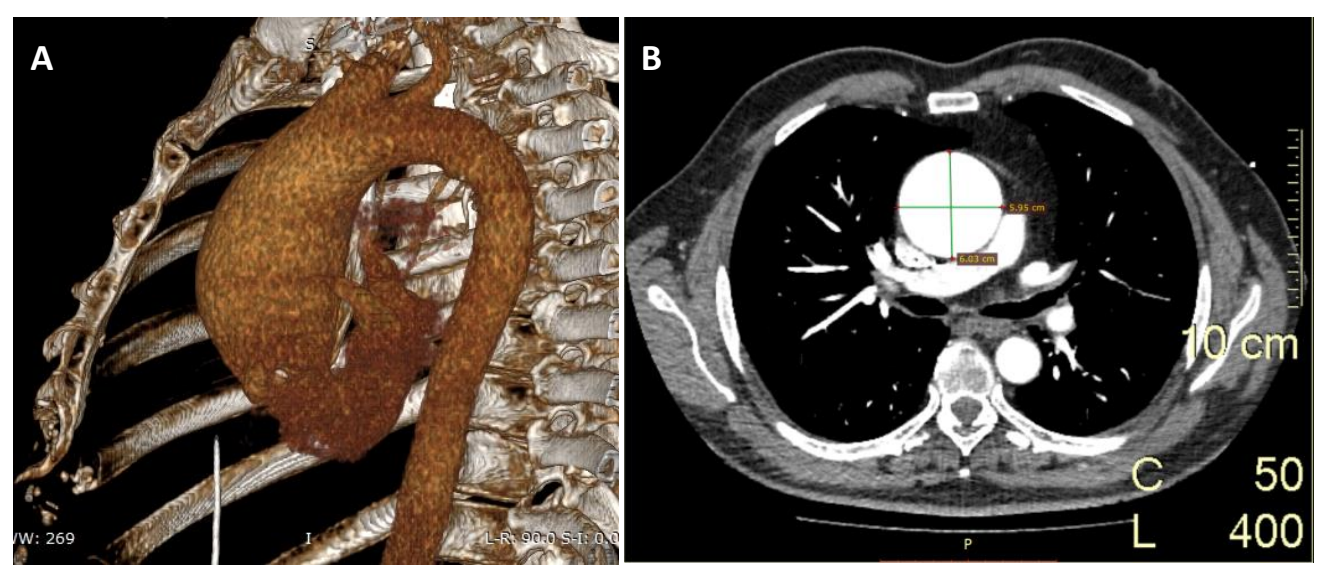


Figure 2

\section{Stage I}

A

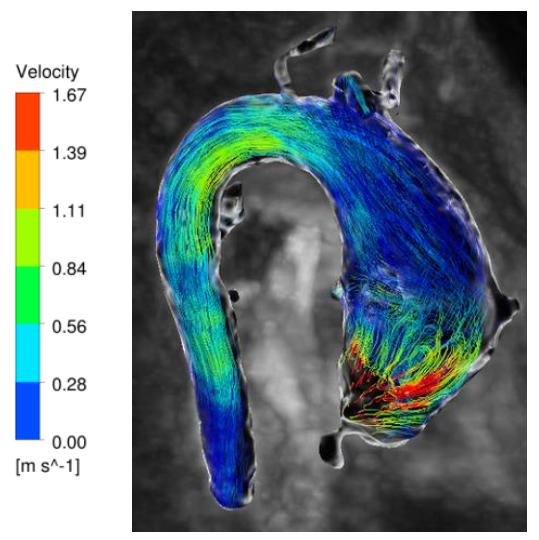

Stage II

\section{D}

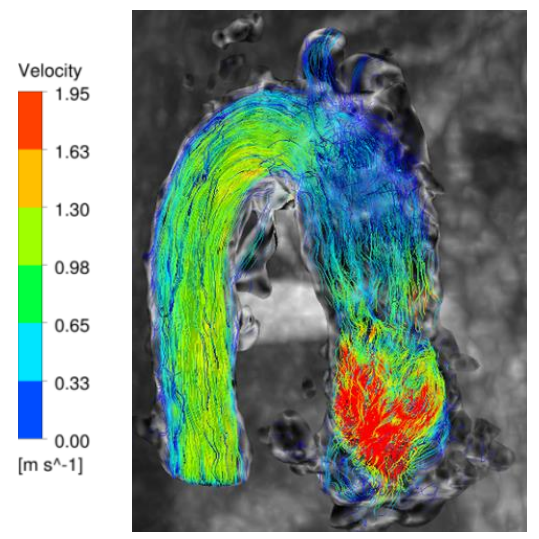

B

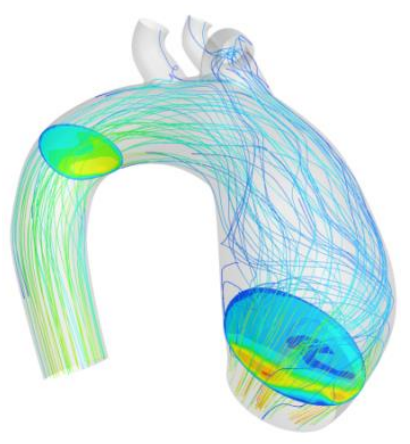

E

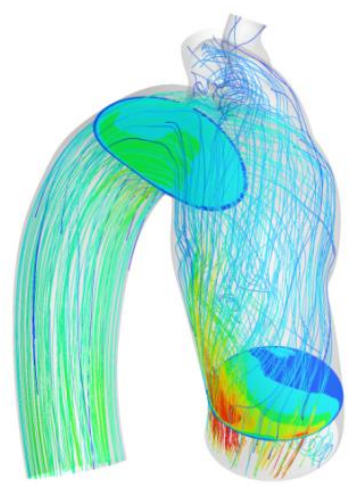

C

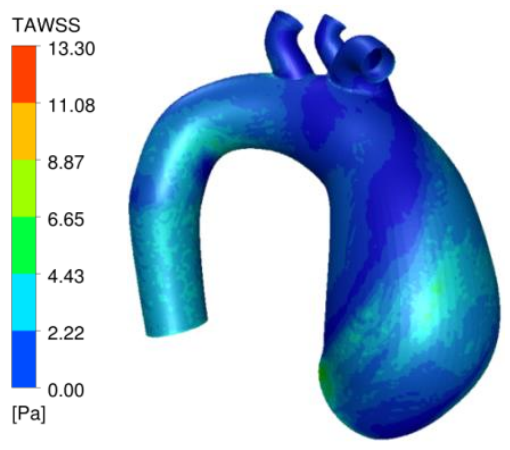

F

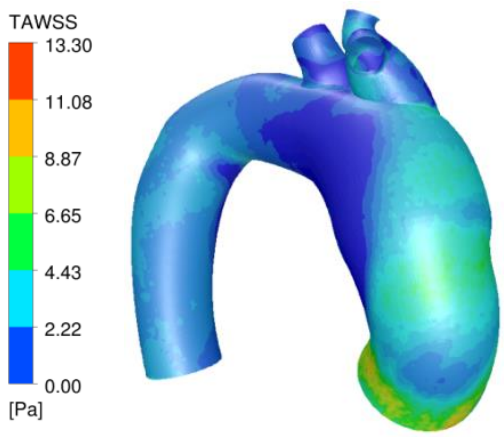

